\title{
FUZZY-BASED FRAMEWORK FOR MANAGING INTER- ENTERPRISE COLLABORATIONS IN PSS CONTEXT
}

\author{
Harrat, Mourad; \\ Belkadi, Farouk; \\ Bernard, Alain \\ Ecole Centrale de Nantes
}

\begin{abstract}
One of the biggest challenges of companies developing new solutions is how to properly manage the different relationships with external partners, especially in the case of Product-Service Systems (PSS). Developing a PSS requires for Original Equipment Manufacturers (OEM) to rethink the organization and roles of the different stakeholders regarding the characteristics of such environments. This paper aims to introduce a methodology to build a framework for inter-enterprise collaboration performance assessment and its corresponding Key Performance Indicators (KPIs). An extensive list of KPIs is established from key factors of collaboration performance and organizational characteristics in PSS context. Finally, further steps of the methodology are discussed where fuzzy techniques are used.
\end{abstract}

Keywords: Product-Service Systems (PSS), Collaborative design, Evaluation, Fuzzy logic

\author{
Contact: \\ Harrat, Mourad \\ Ecole Centrale de Nantes \\ PhD student \\ France \\ mourad.harrat@1s2n.fr
}




\section{INTRODUCTION}

Dealing nowadays with the increasing complexity of products and services, as well as high quality demands forces organisations to collaborate with external partners in order to share efforts, knowledge and skills, so that every partner can focus on his core competences (Benhayoun-sadafiyine 2018; AlHakim and Lu 2017). The diversity of knowledge and capabilities is more important in the case of Product-Service Systems (PSS) offers. In this context, many authors suggest to manufacturers to request service knowledge from foreign suppliers to build a complete solution (Ayala et al. 2017; Bastl et al. 2012; Lockett et al. 2010). To successfully achieve a PSS development project, companies need to reconsider the different coordination routines, roles and scope of competencies regarding the business domain diversity and geographical distribution of stakeholders (Wallin et al. 2011). Regarding this complexity, it is necessary for the organization to follow up and evaluate the performance of the inter-enterprise collaboration, allowing continuous improvements of their processes (Boughzala and De Vreede 2015), and considering the organizational particularities of PSS. Various collaboration assessment models are proposed in literature, as the example of maturity grids (Boughzala and De Vreede 2015; Cuenca et al. 2013; Magdaleno et al. 2011), Bayesian networks and approximate sets (Rosas and Camarinha-Matos 2008), or theory of constraints (Tenera and Rosas 2019). In the PSS context, some works are presented such as readiness assessment of collaborative network organizations for PSS delivery, using conceptual modelling and graph theory (Durugbo and Riedel 2013), and network uncertainty for PSS delivery using fuzzy Delphi-AHP-TOPSIS methods (Wang and Durugbo 2013). However, the literature focusing in collaboration performance within PSS context is still limited and does not consider all factors really influencing the collaboration process. In addition, collaboration in PSS context is generally extended beyond the development stage, where the collaborators during the design phase try to maintain their relationship along the use phase and bring other suppliers since the PSS offer requests complementary efforts to operate.

This paper introduces a new framework to assess inter-enterprise collaboration performance, focusing on the particularities of PSS. For this, we use fuzzy techniques following the process of Ayadi et al. (2013) in his study of assessing trust level in supply chains. In the next section, after introducing the context of our framework, we present the Key Performance Indicators (KPIs) defined as inputs of our assessment model. These KPIs are based on key collaboration performance factors as well as organizational characteristics of PSS business models. Further steps of the methodology are explained in section 3. Finally, we draw a conclusion with some perspectives.

\section{KPIS DEFINED FOR BUILDING THE COLLABORATION MANAGEMENT FRAMEWORK}

In order to achieve performance goals throughout the PSS life-cycle, PSS provider should know how to manage his collaborations. The process presented in Figure 1. covers the PSS development phase, and also the use phase if the same partners from the project are kept.

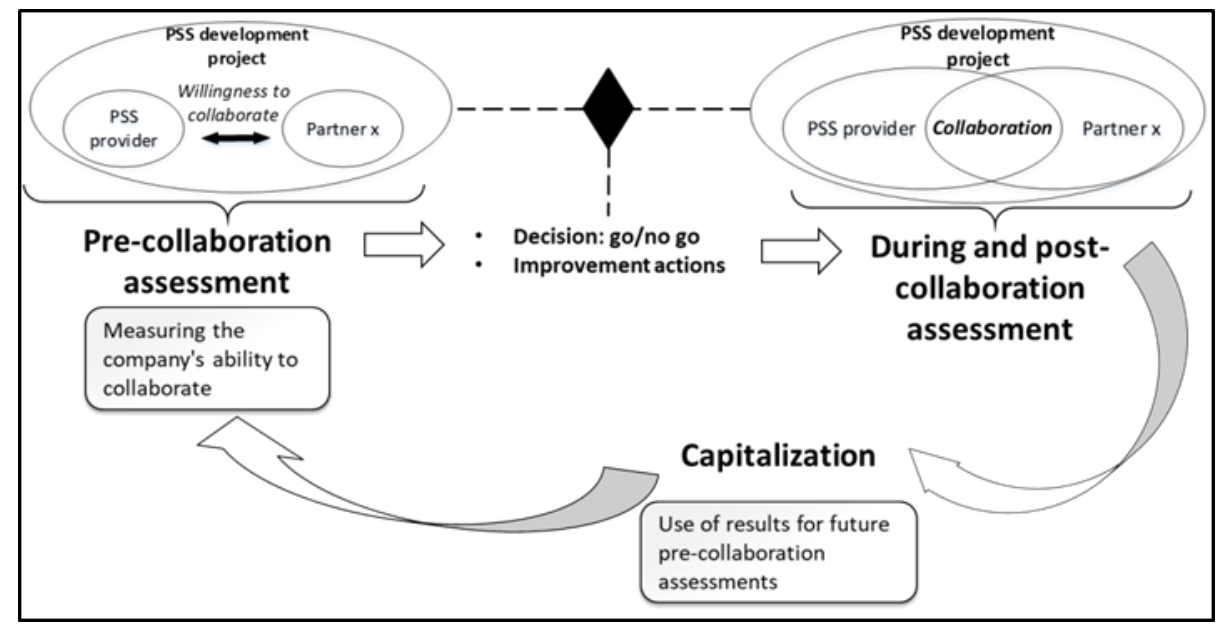

Figure 1. Process of managing inter-enterprise collaboration performance 
Therefore, the ability of the different stakeholders to collaborate in a PSS development project has to be measured in a pre-collaboration assessment. This will lead to the decision to start or no the collaborative project, and a number of improvement actions should be conducted in order to remedy the lacking competences. In further steps of the project, it is necessary to perform an assessment during and post-collaboration, followed by a capitalization step, by using some measures that will be useful for future pre-collaboration assessments. This process (Fig1) contains basic elements that define our collaborations' management framework, which should be generic to different business areas.

The first step of building our framework is to define our measures in the form of KPIs, which we develop in the next sub-sections. This goal is achieved on two complementary points of view. Firstly, since the PSS share some similarities with classical product development in terms of processes, methods and resources, a first category of KPIs seems to be generic (section 2.1). These KPIs are identified based on literature survey regarding collaborative processes in general. Secondly, a dedicated literature survey analysing the key characteristics of PSS paradigm results on the identification of an additional list of specific KPIs (section 2.2).

\subsection{KPls based on key factors of collaboration performance}

Before introducing KPIs to build our assessment model, it is important to identify which factors affect inter-enterprise this performance in an overall context. According to this, Harrat et al. (2020) presented a literature review which addresses the context of inter-firm collaboration between organizations in a general case, and identified a list of ten collaboration performance factors. They suggest that these factors can be adapted to PSS context, as stakeholders of a PSS project are coming originally from conventional business fields. As this latter study matches our framework, we select from their listed factors four of them according to three criteria: (1) their importance in the context of our study, (2) the number of their citations and (3) the ease of measuring them with KPIs. These factors are: trust, commitment, communication quality and coordination. Thus, the KPI building process was based on these factors. First, for trust factor, the proposed KPIs are: pre and post-transaction costs, collaboration history, and knowledge protection.

Transaction costs:

Transaction Costs (TC) are strongly linked to trustworthiness according to Dyer and Chu (2003). These authors defined two types of TC: "ex ante" and "ex post" TC. Ex ante TC are defined as "search and contracting costs". They include the costs generated from searching a desirable partner, negotiating and time spent until agreeing in a contract. In a trusted relationship, negotiation process is more efficient as the buyer is more confident about the authenticity of information given by the partner, and the inclination to guard against opportunistic behaviour is reduced (Dyer and Chu 2003), which will decrease Ex ante TC. On the other hand, ex post TC refer to the costs related to monitoring the agreement and making sure that obligations of each party are respected. Dyer and Chu (2003) found even a stronger relationship between trust and ex post TC, compared to ex ante TC. Indeed, they argued that when trust is high, the parties will spend less time in monitoring the agreement fulfilment or even blaming the other party for problems. Thus, from this study we adapt our definition about KPIs concerning TC as following:

$$
\begin{gathered}
\text { Pre_transaction costs }=\frac{\text { Time spent on negociation }}{\text { total time of transaction }} \\
\text { Post_transaction costs }=\frac{\text { Time spent on monitoring post_transaction problems }}{\text { total time of transaction }}
\end{gathered}
$$

History of collaborations:

Collaborations' history is also an important KPI for trust. Many authors suggest that trust takes time to be developed through long-term business relationships and after several transactions (Dyer and Chu 2010; Belkadi et al. 2016). Longer relationships make partners knowing better each other, and their behaviour become more understandable and predictable (Dyer and Chu 2010). Extensive prior relationship history characterized by successful transactions imply stable relations and a willingness to continue the collaboration for new projects (Belkadi et al. 2016). Therefore, we define the following KPI about History of collaboration: 


\section{History of collaboration \\ $=$ Number of prior collaborations with the concerned partner}

Knowledge protection:

Norman (2002) found that trust significantly reduces knowledge protection supports. She explained that when a company trusts its partner, it will be less dependent to structural mechanisms used to control the information flow and limit the knowledge, as it will rely to the goodwill of the partner who will not use this knowledge in ways that are detrimental to them. On the other hand, an overemphasis on knowledge protection weakens trust and significantly reduces interfirm collaboration (Jean et al. 2014). The metrics used for knowledge protection are the percentage of information sharing, as an adaptation from Norman (2002).

$$
\% \text { of information sharing }=\frac{\text { Quantity of shared information }}{\text { Quantity of available information }}
$$

Where information refers to: marketing plans, technical information, and strategic information.

The second factor "commitment", is related to the KPIs: participation, schedule respect and reactivity.

\section{Participation:}

On a perspective of commitment, Westphal et al. (2007) proposed the degree of participation in meeting or conference calls as a metric for collaboration performance. Participation refers to the active involvement of different parties in planning, problem solving, and decision-making (Wibisono et al. 2018). Empirically, Lee and Kim (1999) found that participation was significantly related to partnership quality, and positively associated with trust, business understanding, and commitment. We adapt this indicator by taking into account partner's participation in the project decisions, in order to consider efficiency of participation, as follows:

$$
\% \text { of participation }=\frac{\text { Number of decisions involving partner }}{\text { Total number of taken decisions }}
$$

Schedule respect:

Another metric in the perspective of commitment presented by Westphal et al. (2007) is concerning deviations from planning. When a partner puts the project in his priorities, he will give importance to respecting time schedules and delivery dates. In particular, parties who exchange high-quality knowledge and are fully committed to the project will be able to avoid delays and reworks (Yan and Dooley 2014) . Thus, we adapt this indicator from Westphal et al. (2007) as following:

$$
\text { Schedule respect }=\frac{\text { Number of deviations from planning }}{\text { Total number of planned actions }}
$$

Reactivity:

This metric was defined by Cheriti (2011) to indicate responsiveness of suppliers to different buyer's requests. Reactivity of partners to different other parties' requests is one of signs of commitment and the importance given to the corresponding project. Accordingly, a close and strong relationship with a supplier will enhance his responsiveness (Davis-Sramek et al. 2019). Thus, reactivity indicator is calculated as following:

$$
\text { Average response time }=\frac{\text { Sum of the delays of the different requests }}{\text { Total number of requests }}
$$

The third factor is communication quality. Its associated KPIs are: effectiveness of meetings and openness of communication channels.

Effectiveness of meetings:

Some indicators were proposed by Wallin et al. (2011) in order to measure both quantity and quality of collaboration activities or connections (e.g. meetings). Because it is not enough to measure only the quantity, we need also to explore the quality of collaboration meetings in order to know if they involve collaboration ideation or only exchange of information (Wallin et al. 2011), which will gives us an idea about their effectiveness. Therefore, we propose a ratio to calculate the effectiveness of meetings:

Effectiveness of meetings $=$

Number of meetings conducting to ideation or decision making

Total number of meetings 
Openness of communication channels:

Limitation of communication channels is another way to protect knowledge. A less restricted communication channels is a sign of a good level of trust (Norman 2002), and will positively impact on knowledge sharing. Identically to knowledge protection indicator, we measure openness of communication channels as following:

$$
\begin{aligned}
& \text { Openness of communication channels } \\
& \qquad=\frac{\text { Number of accessible communication channels }}{\text { Number of existing communication channels }}
\end{aligned}
$$

The fourth factor is coordination. The KPIs identified to be related to this factor are: planning adjustment and conflict solving.

Planning adjustment:

Westphal et al. (2007) proposed "frequency of adjusting/coordinating the planning" as an indicator related to decision synchronization in Virtual Organizations. Adjusting planning will improve coordination between the partners in the project, by avoiding decision conflicts and task duplication. In the event of technology issues, when collaboration partners can no longer focus on formally agreed upon milestones, they must adjust milestones and target dates to ensure adherence to schedules (Pemartín and Rodríguez-Escudero 2020). This KPI is defined so:

\section{Frequency of planning adjustment $=$ Number of planning adjustments per month}

Conflict solving:

Resolving conflicts is essential for collaborative projects and has a positive impact on collaboration quality (Dietrich et al. 2010). A high level of communication willingness will help project teams to coordinate and resolve conflicts which in turn contribute towards the project success (Wu et al. 2017). Thus, conflicts are solved effectively in a team level and do not need an involvement of the top management. As an adaptation from Westphal et al. (2007), we measure the number of problems that could not be solved but need escalation to direction.

$$
\text { Conflict solving }=\frac{\text { Number of problems needing escalation to direction }}{\text { Total number of encountred problems }}
$$

The proposed indicators build from the most important collaboration factors (Harrat et al. 2020) are necessary, but not sufficient to assess inter-enterprise collaboration performance for PSS development. Indeed, we need also to explore the PSS organizational context in order to highlight its particularities and collaboration practices, from where we can define new KPIs to build a more extensive framework.

\subsection{KPIs based on organizational practices within PSS context}

The second type of KPIs concerns organizational practices and suggestions which characterize interenterprise collaboration in PSS context. From a selection of 10 articles, PSS organizational practices were proposed by authors through different terms : routines (Wallin et al. 2015), characteristics (Saccani et al. 2014), tactics and operational practices (Reim et al. 2015)...etc. From this small literature review, we could identify some practices and transformed them into KPIs. We present these practices under five main themes: information and knowledge sharing, interoperability and adaptation, contract management, whole cycle-life consideration, and customers interaction data.

Information and knowledge sharing:

According to Bastl et al. (2012), servitisation (i.e. the process to transform companies into PSS providers) context needs higher levels of integration of the supply chain. Therefore, information exchange should be open, bidirectional with higher frequency comparing to the context of pure products. Accordingly, Lockett et al. (2010) suggest that the adoption of PSS business models requires a more intensive information exchange between supply chain members, which implies the necessity to have closer relationships. In the light of these suggestions, we propose two KPIs related to information and knowledge sharing, which are both frequency and reciprocity of information/knowledge sharing:

Frequency of sharing = Quantity of knowledge sharing activities per month

$$
\text { Reciprocity of sharing }=\frac{\text { Quantity of shared knowledge by the party }}{\text { Total quantity of shared knowledge }}
$$


Interoperability and adaptation:

One of the definitions of interoperability is the ability of companies to interact with each other. Interoperability is achieved if the interaction can at least take place on three levels: data level, application level and business process level (IDEAS 2003). In PSS context, linkages and interactions between systems, procedures, and routines should be more formalized and closely coupled comparing to pure products development environment (Bastl et al. 2012). These operational links which facilitate processes and increase their effectiveness, can be implemented the form of integrated information systems that allow parties to exchange schedules, request spare parts, or provide feedbacks on interventions (Saccani et al. 2014). Furthermore, it is suggested that some adaptations to partners' needs are more necessary in the case of PSS business models, with the need of a reciprocity from partners in these adaptations (Bastl et al. 2012), or even the creation of common processes to manage interdependencies between partners and support interactions between them (Windahl and Lakemond 2006). According to these suggestions, we define the following KPIs: the percentage of mutual adaptation to the mode of operation (processes, procedures, practices...) between the partners, as well as the percentage of adequacy of the standards used by both parties:

$$
\begin{gathered}
\% \text { of mutual adaptation }=\frac{\text { Number of aligned processes and practices }}{\text { Total number of processes and practices }} \\
\% \text { of adequacy of standards }=\frac{\text { Number of common standards }}{\text { Total number of used standards }}
\end{gathered}
$$

\section{Contract management:}

Concerning contractual mechanisms, Bastl et al. (2012) highlight that contracts in PSS context should be more detailed in order to capture the high complexity and risk levels in servitized environments, or otherwise to complete contracts with relational mechanisms based on trust. For example, promoting a win/win mentality. Based on a literature review, Reim et al. (2015) presented some PSS tactics on key aspects of contracts: Responsibility and terms of agreements, formalization and complexity, and risk level. These aspects are presented according to each PSS type (i.e. Product-Oriented, PO; UsageOriented, UO; and Result-Oriented, RO). Contracts are more complex in the case of PSS comparing to pure products, but it differs from one PSS type to another (Richter and Steven 2009). Indeed, Reim et al. (2015) found complexity lower for PO, medium of UO and higher for RO. This is also the case for risk level for PSS business models, where they are generally related to gains received by the PSS provider, and specifically to higher efforts expected to fulfil the agreement in case of PO, adverse customer behaviour in case of UO, and results delievery in case of RO (Reim et al. 2015). In this regard, complexity and PSS type are introduced in our model as attributes in order to consider the project's context. We also introduce in a KPI for risk level which is related to partner's commitment:

$$
\begin{aligned}
& \% \text { of contractually commited risk } \\
& \qquad=\frac{\text { Amount of commited risk by the party }}{\text { Total amount of commited risk by all the parties }}
\end{aligned}
$$

Whole life-cycle consideration:

Another important characteristic of PSS, is the necessity of organizations to consider the whole life-cycle of the solution when developing a PSS, because development and delivery processes are extended beyond the sale of the product (Wallin et al. 2015b37). It implies the necessity for firms to understand the entire PSS life-cycle, and to be able to evaluate its related costs and risks (Lockett et al. 2010).

This can include the involvement of maintenance and after-sales services, engineering department and marketing functions in the early stages of the development process (Lockett et al. 2010; Resta et al. 2017). It is therefore necessary to involve all supply chain partners in order to perform a complete evaluation of the offer (Wallin et al. 2015). Lockett et al. (2010) mentioned that this perspective can present certain difficulties. In some cases, costs of replacing parts can be lower than those of repairing and maintenance, taking into accounts the value of raw material and the time taken to repair. Difficulties to evaluate the life-cycle costs of the PSS and risks due to an incorrect estimation are also some obstacles to adopt this perspective (Lockett et al. 2010). These difficulties were explored by authors who suggest an adapted design process of the solutions throughout their life-cycle (Aurich et al. 2006), as well as design for maintenance or for services in PSS development (Apitz et al. 2013). In the light of these suggestions, we introduce a KPI which concern the involvement during the development phase of the 
various departments present throughout the PSS life-cycle (marketing, engineering, manufacturing, maintenance and after-sales service):

$\%$ of department involvement in early design $=$ Number of participations of the department in early design activities

Total number of early collaborative design activities

Customers' interaction data:

Interactions between the provider and his customers generally increase when the firms are service oriented (Reim et al. 2015). Indeed, customer relationship needs a higher attention in the PSS context (Wallin et al. 2015). As mentioned by Reim et al. (2015), customer interactions differs in intensity depending on the type of PSS. They are regular in intensity for PO and higher for UO because of the need to ensure the usability of the PSS. The intensity of these interactions increases even more for RO regarding the need to keep a continuous contact with the customer (Reim et al. 2015). PSS, by their nature, provide the ability to collect customer data during its operation or use. Thus, the authors recommend in this context the creation of a customer relationship platform accompanied by a regular collection of customer needs (Wallin et al. 2015; Reim et al. 2015; Saccani et al. 2014; Avlonitis et al. 2013; Windahl and Lakemond 2006). The metric we introduce express the percentage of data collection actions compared to the number of interactions.

$$
\% \text { of data collection }=\frac{\text { Number of data collection action }}{\text { Total number of customer interactions }}
$$

\section{A METHODOLOGY TO BUILD A FRAMEWORK FOR COLLABORATION PERFORMANCE ASSESSMENT AND MANAGEMENT}

In order to build our framework for inter-enterprise collaboration performance assessment and management, we use fuzzy logic techniques, regarding its capacities to counter ambiguity and imprecision problems in calculations (Wang and Durugbo 2013), especially in solving socioeconomic problems. In our case, we have qualitative outputs (e.g. trust, commitment...etc.) which have no particular units of measure. Using fuzzy techniques is one solution to deal with this issue by using linguistic terms instead of precise numerical values. The considered inputs of this model at the fuzzification stage are our KPIs defined before. Each KPI is represented by a Membership Function (MF). Then, the system outputs are collaboration performance factors (Figure 2).

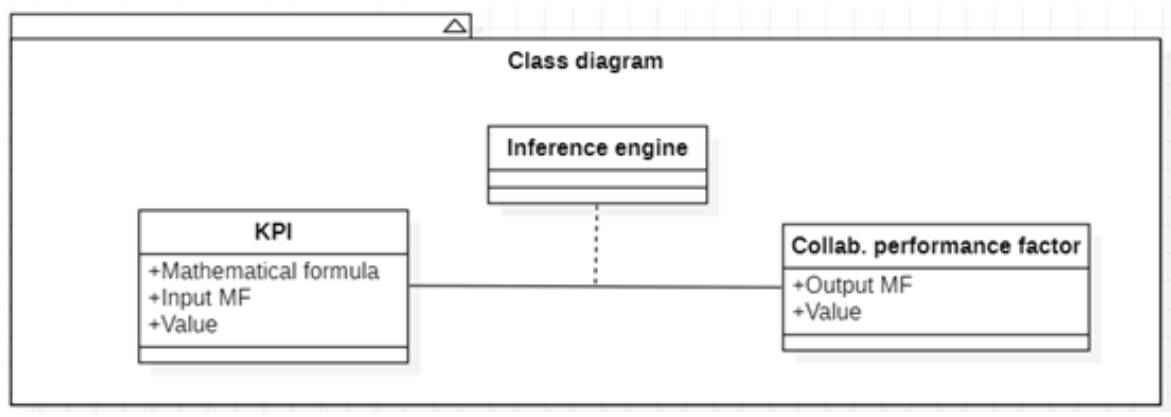

Figure 2. Fuzzy logic system for collaboration performance assessment

The proposed methodology is inspired from Ayadi et al. (2013) who used fuzzy techniques to assess trust level between partners is supply chain. To illustrate our work, we present an example of the KPI input "Pre-transaction costs" which is related to the output "trust factor" (Figure 3).

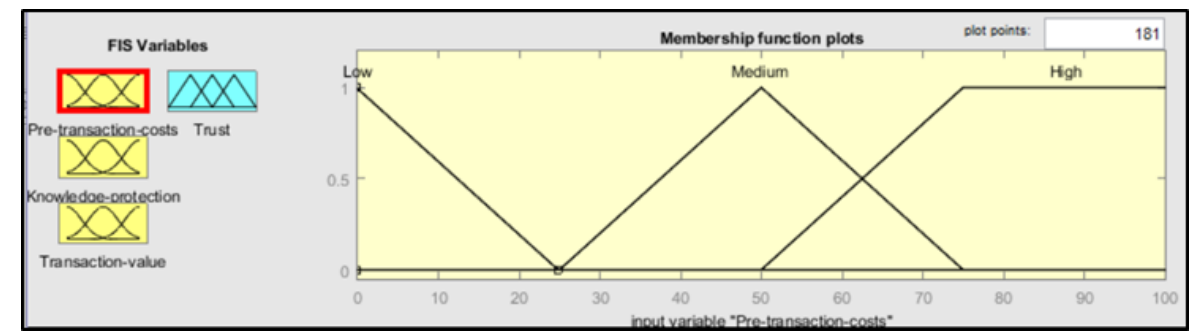

Figure 3. Example of membership function "pre-transaction costs" 
We chose a mix of triangular and trapezoidal MF and standard thresholds for limit values of the MFs $(0 \%, 25 \%, 50 \%, 75 \%$ and $100 \%)$ to cover large possibilities. Three MFs are defined to describe the value of transaction costs: Low $(0,0,25)$, medium $(25,50,75)$ and high $(50,75,100,100)$. The MFs "low" and "medium" are triangular, based on suggestions of Ayadi et al. (2013) who consider this form appropriate to represent human factors and widely used in literature. On the other hand, the MF "high" is trapezoidal as we consider that from a percentage of negotiation time higher or equal to 75\% of transaction time, the costs are high with a fuzzy value $=1$ (certain).

The next step is the creation of inference rules. For this step, the Mamdani's fuzzy inference method is used as it is convenient for human inputs (Sivanandam et al. 2007). Different methods can be used to generate fuzzy rules, such as the involvement of experts by using semi-structured interviews (Ayadi et al. 2013). In the proposed approach, we opt for using a structured questionnaire to collect experts' points of view. Then, the inference rules will be generated from a decision tree, created by the C4.5 learning system, as suggested by Hall and Lande (1998). Indeed, these authors mentioned that decision tree theory provides a well-understood mechanism for inducing classification rules from data. To do so, it is necessary to split the continuous inputs and outputs into classes, as we did for Pre-transaction costs (three MF: low, medium and high TC). Using data collected from an online survey, we select the KPIs as attributes and factors (e.g. trust) as the target variable. To create fuzzy rules, we perform a depth search in the decision tree, and each time a path reaches a leaf, a rule is created with the previous nodes by combining them with the "and" (Hall and Lande 1998). the following rule is formulated as an example in figure 4:

IF Risk shared = lower (from partner) AND Planning respect $=$ total AND Participation $=($ Very High OR High OR Medium $)$ THEN Commitment $=$ High.

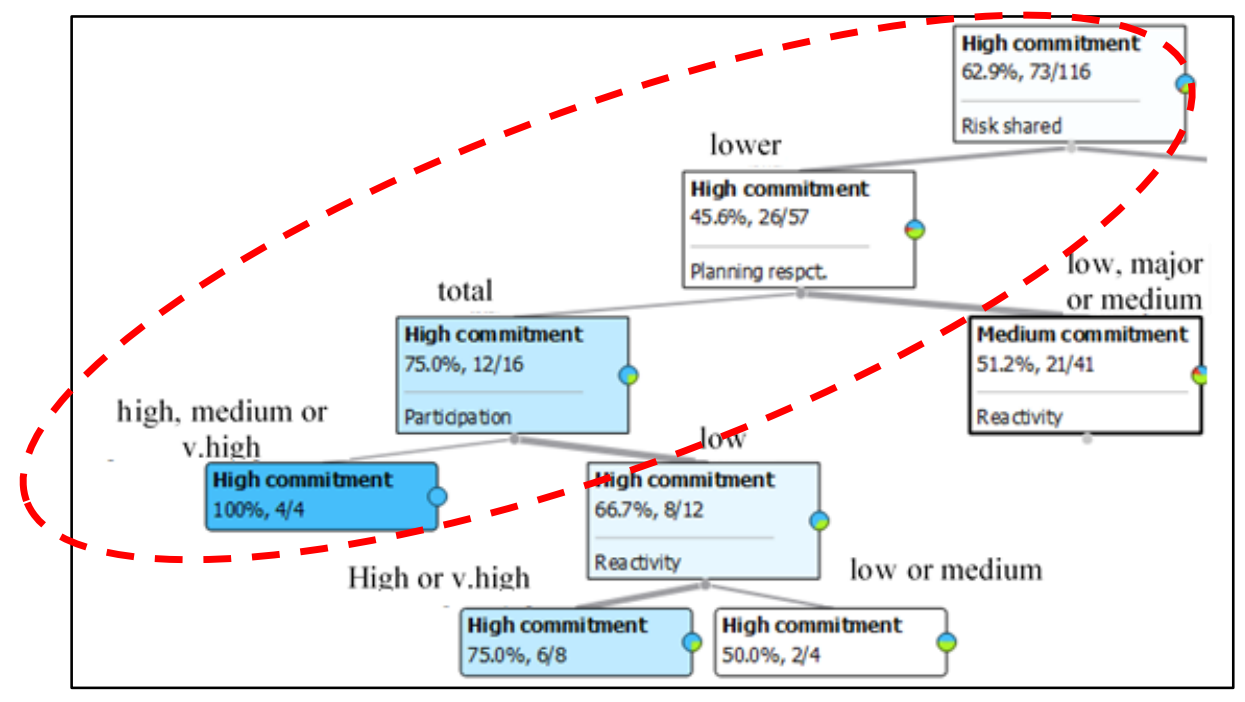

Figure 4. Example of decision tree used for fuzzy rules generation

The rules can be directly used, or may be further tuned to increase their reliability (Hall and Lande 1998). For this goal, it is also needed to pay attention to the population responses' number and percentage (mentioned in each node) which match the rule. A post-pruning approach can also be performed in order to remove irrelevant branches from the tree, in addition to the possibility for the expert to intervene in the process of model creation in order to get a better interpretation and understandability of the results (Rakotomalala 2005).

\section{CONCLUSION AND PERSPECTIVES}

The objective of this paper is twofold. First, it aims to present the proposed KPIs as necessary to assess inter-enterprise collaboration performance in the context of PSS development. On the other hand, it introduces the early steps of a methodology of building a framework for collaboration performance management. Further steps after the development of the decision support system, are the confrontation of the overall model to real case studies. Several case studies in various business areas are in progress. One of them concerns the development of connected shoes, where deep interviews have been realized with different experts from the involved companies to extract business rules. The ongoing step is to present 
and deeply discuss the whole model. The objective of this model discussion is to perform some adjustments of the framework regarding the suggestions coming from industrials. Interviews are being performed about the relevance of the membership functions, KPIs, inference rules and the overall consistency of the assessment system.

\section{REFERENCES}

Al-Hakim, L., and W. Lu, 2017: The role of collaboration and technology diffusion on business performance. Int. J. Product. Perform. Manag., 66, 22-50, https://dx.doi.org/10.1108/IJPPM-08-2014-0122.

Apitz, N., T. C. McAloone, A. G. i Mateu, J. A. B. Andersen, K. Mougaard, L. M. Neugebauer, and J. Hsuan, 2013: PSS Organisation: A workbook in the PROTEUS Series. Technical University of Denmark, 52 p.

Aurich, J. C., C. Fuchs, and C. Wagenknecht, 2006: Life cycle oriented design of technical Product-Service Systems. J. Clean. Prod., 14, 1480-1494, https://dx.doi.org/10.1016/j.jclepro.2006.01.019.

Avlonitis, V., J. Hsuan, T. C. McAloone, A. G. i Mateu, J. B. Andersen, K. Mougaard, L. Neugebauer, and T. Ahm, 2013: PSS Readiness Manual: A workbook in the PROTEUS series. 43 p.

Ayadi, O., N. Cheikhrouhou, and F. Masmoudi, 2013: A decision support system assessing the trust level in supply chains based on information sharing dimensions. Comput. Ind. Eng., 66, 242-257, https://dx.doi.org/10.1016/j.cie.2013.06.006.

Ayala, N. F., C. A. Paslauski, A. Ghezzi, and A. G. Frank, 2017: Knowledge sharing dynamics in service suppliers' involvement for servitization of manufacturing companies. Int. J. Prod. Econ., 193, 538-553, https://dx.doi.org/10.1016/j.ijpe.2017.08.019.

Bastl, M., M. Johnson, H. Lightfoot, and S. Evans, 2012: Buyer-supplier relationships in a servitized environment: An examination with Cannon and Perreault's framework. Int. J. Oper. Prod. Manag., 32, 650-675, https://dx.doi.org/10.1108/01443571211230916.

Belkadi, F., M. Messaadia, A. Bernard, and D. Baudry, 2016: Collaboration management framework for OEM suppliers relationships: a trust-based conceptual approach. Enterp. Inf. Syst., 11, 1-25, https://dx.doi.org/10.1080/17517575.2016.1250166.

Benhayoun-sadafiyine, L., 2018: La capacité d'absorption des PMEs intégrées dans des réseaux d'innovation collaboratifs : évaluation à travers une grille de maturité.

Boughzala, I., and G. J. De Vreede, 2015: Evaluating Team Collaboration Quality: The Development and Field Application of a Collaboration Maturity Model. J. Manag. Inf. Syst., 32, 129-157, https://dx.doi.org/10.1080/07421222.2015.1095042.

Cheriti, S., 2011: Conception collaborative : propositions pour construire et piloter des relations performantes avec les fournisseurs. https://dx.doi.org/10.1081/Pln-200055550.

Cuenca, L., A. Boza, M. M. E. Alemany, and J. J. M. Trienekens, 2013: Structural elements of coordination mechanisms in collaborative planning processes and their assessment through maturity models: Application to a ceramic tile company. Comput. Ind., 64, 898-911, https://dx.doi.org/10.1016/j.compind.2013.06.019.

Davis-Sramek, B., A. Omar, and R. Germain, 2019: Leveraging supply chain orientation for global supplier responsiveness: The impact of institutional distance. Int. J. Logist. Manag., 30, 39-56, https://dx.doi.org/10.1108/IJLM-09-2017-0225.

Dietrich, P., P. Eskerod, D. Dalcher, and B. Sandhawalia, 2010: The dynamics of collaboration in multipartner projects. Proj. Manag. J., 41, 59-78, https://dx.doi.org/10.1002/pmj.20194.

Durugbo, C., and J. C. K. H. Riedel, 2013: Readiness assessment of collaborative networked organisations for integrated product and service delivery. Int. J. Prod. Res., 51, 598-613, https://dx.doi.org/10.1080/00207543.2012.658529.

Dyer, J. H., and W. Chu, 2003: The role of trustworthiness in reducing transaction costs and improving performance: Empirical evidence from the United States, Japan, and Korea. Organ. Sci., 14, 57-68, https://dx.doi.org/10.1287/orsc.14.1.57.12806.

Dyer, and Chu, 2010: The determinants of trust in supplier-automaker relationships in the U.S. Japan and Korea. J. Int. Bus. Stud., 31, 259-285. http://dx.doi.org/10.1057/jibs.2010.34.

Hall, L. O., and P. Lande, 1998: Generation of Fuzzy Rules from Decision Trees. J. Adv. Comput. Intell. Intell. Informatics, 2, 128-133, https://dx.doi.org/10.20965/jaciii.1998.p0128.

Harrat, M., F. Belkadi, and A. Bernard, 2020: Towards a modelaing framework of collaboration in PSS development project: A review of key factors. Procedia CIRP, 90, 736-741, https://dx.doi.org/10.1016/j.procir.2020.01.130.

IDEAS, 2003: IDEAS Project Deliverables (WP1-WP7), Public Reports, 2003.

Jean, R. J. B., R. R. Sinkovics, and T. P. Hiebaum, 2014: The effects of supplier involvement and knowledge protection on product innovation in customer-supplier relationships: A study of global automotive suppliers in China. J. Prod. Innov. Manag., 31, 98-113, https://dx.doi.org/10.1111/jpim.12082.

Lee, J.-N., and Y.-G. Kim, 1999: Effect of Partnership Quality on IS Outsourcing Success: Conceptual Framework and Empirical Validation. J. Manag. Inf. Syst., 15, 29-61, https://dx.doi.org/10.1080/07421222.1999.11518221. 
Lockett, H., M. Johnson, S. Evans, and M. Bastl, 2010: Product Service Systems and supply network relationships: an exploratory case study. J. Manuf. Technol. Manag., https://dx.doi.org/10.1108/IJMF-042016-0078.

Magdaleno, A. M., R. M. De Araujo, and C. M. L. Werner, 2011: A roadmap to the Collaboration Maturity Model (CollabMM) evolution. Proc. 2011 15th Int. Conf. Comput. Support. Coop. Work Des. CSCWD 2011, 105-112, https://dx.doi.org/10.1109/CSCWD.2011.5960062.

Norman, P. M., 2002: Protecting knowledge in strategic alliances resource and relational characteristics. J. High Technol. Manag. Res., 13, 177-202, https://dx.doi.org/10.1016/S1047-8310(02)00050-0.

Pemartín, M., and A. I. Rodríguez-Escudero, 2020: Is the formalization of NPD collaboration productive or counterproductive? Contingent effects of trust between partners. BRQ Bus. Res. Q., 24, 2-18, https://dx.doi.org/10.1177/2340944420916307.

Rakotomalala, R., 2005: Arbres de decision. Revue Modulad.

Reim, W., V. Parida, and D. Örtqvist, 2015: Product-Service Systems (PSS) business models and tactics - A systematic literature review. J. Clean. Prod., 97, 61-75, https://dx.doi.org/10.1016/j.jclepro.2014.07.003.

Resta, B., P. Gaiardelli, S. Cavalieri, and S. Dotti, 2017: Enhancing the Design and Management of the ProductService System Supply Chain: An Application to the Automotive Sector. Serv. Sci., 9, 302-314, https://dx.doi.org/10.1287/serv.2017.0193.

Richter, A., and M. Steven, 2009: On the relation between industrial product-service systems and business models. Operations Research Proceedings 2008, Springer, 97-102.

Rosas, J., and L. M. Camarinha-Matos, 2008: Modeling collaboration preparedness assessment. Collab. Networks Ref. Model., 227-252, https://dx.doi.org/10.1007/978-0-387-79426-6_17.

Saccani, N., F. Visintin, and M. Rapaccini, 2014: Investigating the linkages between service types and supplier relationships in servitized environments. Int. J. Prod. Econ., 149, 226-238, https://dx.doi.org/10.1016/j.ijpe.2013.10.001.

Sako, M., 1991: The Role of Trust in Japanese buyer-supplier relationships. Ric. Econ., 449-473. https://pascalfrancis.inist.fr/vibad/index.php?action=getRecordDetail\&idt=6556575.

Sivanandam, S. N., S. Sumathi, and S. N. Deepa, 2007: Introduction to fuzzy logic using MATLAB. 1-430 pp.

Tenera, A., and J. Rosas, 2019: Collaborative Networks Management from a Theory of Constraints Perspective. 2, 271-282, https://dx.doi.org/10.1007/978-3-030-28464-0_24.

Wallin, J., A. Larsson, O. Isaksson, and T. Larsson, 2011: Measuring Innovation Capability - Assessing Collaborative Performance in Product-Service System Innovation. Functional Thinking for Value Creation, Springer Berlin Heidelberg, Berlin, Heidelberg, 207-212 http://link.springer.com/10.1007/978-3-64219689-8_37 (Accessed April 8, 2019).

Wallin, V. Parida, and O. Isaksson, 2015: Understanding product-service system innovation capabilities development for manufacturing companies. J. Manuf. Technol. Manag., 26, 763-787, https://dx.doi.org/10.1108/JMTM-05-2013-0055.

Wang, X., and C. Durugbo, 2013: Analysing network uncertainty for industrial product-service delivery: A hybrid fuzzy approach. Expert Syst. Appl., 40, 4621-4636, https://dx.doi.org/10.1016/j.eswa.2013.01.062.

Westphal, I., K. D. Thoben, and M. Seifert, 2007: Measuring collaboration performance in virtual organizations. IFIP International Federation for Information Processing, Vol. 243 of, Springer US, Boston, MA, 33-42 http://link.springer.com/10.1007/978-0-387-73798-0_4 (Accessed May 22, 2018).

Wibisono, Y. Y., R. Govindaraju, D. Irianto, and I. Sudirman, 2018: Interaction quality and the influence on offshore IT outsourcing success. Proc. 2017 Int. Conf. Data Softw. Eng. ICoDSE 2017, 2018-Janua, 1-6, https://dx.doi.org/10.1109/ICODSE.2017.8285879.

Windahl, C., and N. Lakemond, 2006: Developing integrated solutions: The importance of relationships within the network. Ind. Mark. Manag., 35, 806-818, https://dx.doi.org/10.1016/j.indmarman.2006.05.010.

Wu, G., C. Liu, X. Zhao, and J. Zuo, 2017: Investigating the relationship between communication-conflict interaction and project success among construction project teams. Int. J. Proj. Manag., 35, 1466-1482, https://dx.doi.org/10.1016/j.ijproman.2017.08.006.

Yan, T., and K. J. Dooley, 2014: Buyer-supplier collaboration quality in new product development projects. $J$. Supply Chain Manag., 50, 59-83, https://dx.doi.org/10.1111/jscm.12032. 